\title{
Correlations of electrocardiographic pattern, shape of ventricular septum, and isovolumetric relaxation time in irregular hypertrophic cardiomyopathy (obstructive cardiomyopathy)
}

\author{
Dietrich Harmjanz, Dedo Böttcher, and Günter Schertlein \\ From the Department of Internal Medicine, Arbeitsgruppe für klinische Kardiologie, \\ Medizinische Hochschule, Hannover, West Germany
}

The anatomical structures of the ventricles, and especially the ventricular septum, are characterized by an irregular hypertrophy. Patients with a predominantly circumscribed hypertrophy of the ventricular septum exhibit pathological $Q$ waves in their chest leads, whereas those with a predominantly diffuse septal hypertrophy show delta waves in the chest leads. A loss of ventricular compliance is a common finding. Patients with localized hypertrophy have a shorter relaxation period than those with a diffuse hypertrophy. Delta waves and pathological $Q$ waves represent local pre-excitation in these patients.

Irregular hypertrophic cardiomyopathy, a term we proposed for obstructive cardiomyopathy (Harmjanz et al., 1967b), is still a poorly understood disease of the heart muscle, though it has been studied for more than ro years.

The results of different authors (Braunwald et al., 1964; Menges, Brandenburg, and Brown, I96I; Gorlin et al., I964) reveal the haemodynamics of this disease to be variable and to be of little value as a parameter for correlations.

It is the purpose of this paper to draw attention to correlations between morphology and function in irregular hypertrophic cardiomyopathy apart from the otherwise impressive haemodynamics (Ross et al., 1966).

\footnotetext{
Material and methods

Twenty-five patients ( 16 men, 9 women), mean age 31 years, were examined. Physical status, 12/13 lead electrocardiogram, phonocardiogram, and apex cardiogram, $x$-ray examination in two planes, and catheterization of the left and the right heart were performed in every case. In 13 of the 25 patients biplane angiocardiograms of the right and left ventricle were performed within an interval of a few minutes. To avoid errors of parallax we ensured that the position of the patient was identical during the two series. We took the outlines of the left and right ventricles, respectively, from the angiocardiograms. The outlines

Received 2 March 1971.
}

were superimposed in order to get an impression of the form of the ventricular septum. Angiocardiograms of the diastolic and systolic phases were evaluated separately. All electrocardiographic, phonocardiographic, and mechanocardiographic studies were performed either on direct writing- or photo-multichannel-recorders. All channels were tested thoroughly for coincidence.

\section{Results}

Haemodynamics Pressure gradients between the apex of the right ventricle and the cavity of the right ventricle ranging from $\mathrm{I}_{4}-$ I20 $\mathrm{mmHg}$ were found in 14 patients. A pressure gradient exceeding $8 \mathrm{mmHg}$ between the right ventricular cavity and the pulmonary artery was recorded in 7 patients. An intraventricular gradient in the left ventricle ranging from $30-185 \mathrm{mmHg}$ was recorded in 12 patients. The highest incidence (20 patients) in pressure gradients was found between the apex of the left ventricle and the aorta (range 30-190 $\mathrm{mmHg}$ ). The left atrial mean pressure was normal in 5 of 22 patients, reached the upper limit value (10 $\mathrm{mmHg}$ ) in 5 patients, and exceeded $10 \mathrm{mmHg}$ in $12 \mathrm{pa}$ tients. The left atrial ' $a$ ' wave was normal in 3 patients and was higher than $13 \mathrm{mmHg}$ in the other 19 patients.

Angiocardiograms The features of the ventricular septum were found to be altered in two ways: by predominantly circumscribed 
hypertrophy (Group I) or by predominantly diffuse hypertrophy (Group II).

Group I Predominantly circumscribed hypertrophy was assumed when the left ventricle showed an indentation on the lower margin in diastole and more obviously in systole. This indentation has the form of a semicircle, its zenith lying at half the distance from the apex to the aortic valve area. The longitudinal axis of the ventricles is broken by the circumscribed hypertrophy of the septum. Half shadows distal and proximal from the zenith give the left ventricle the form of a corkscrew (Fig. 2, Case I). The apex of the left ventricle appears to be neither deformed nor shifted. The left margin of the right ventricle is shifted to the right and exhibits, in some cases, a concave left border like a half-moon. The apex is well preserved and not or a little shifted to the right. The crucial point of circumscribed hypertrophy of the ventricular septum is that the hypertrophy is localized in the middle of the ventricular septum and does not affect the position of the apexes of both ventricles in diastole (Fig. I, Cases I, 2, and 3). In systole a gap between the left and the right ventricle becomes apparent. This gap is

" formed by the predominantly localized hypertrophy of the ventricular septum.

Group II Predominantly diffuse hypertrophy of the ventricular septum was assumed when the apexes of both ventricles did not lie close together in the anteroposterior plane in diastole.

Subgroup IIa In some patients the form of the left ventricle in diastole is that of a cone. The longitudinal axis is straight. The more the left ventricle had this form the thicker the septum appeared to be in the apical region (Fig. I, Cases 4, 5, 6, 7, and 8). In systole the left ventricle in the 2 patients (Cases 7

and 8) seems to be grossly deformed and the cavities are divided into several compartments.

Subgroup IIb In some patients (Cases 9, 10, II, and I2) the left ventricular longitudinal axis is broken as it is in cases with localized hypertrophy of the ventricular septum, but the apexes of both ventricles lie remote from one another even in diastole. In one patient (Case 13) the deformation of the left ventricular cavity was so much advanced that no classification into these two subgroups was possible.

The right ventricle forms the counterpart. The left border of the right ventricle is shifted to the right. The outlines give little information as to the problem of localized or diffuse hypertrophy of the ventricular septum.

The crucial point of predominantly diffuse hypertrophy of the ventricular septum is that the septum separates widely the apexes of both ventricles in diastole. In systole in mild cases (Cases 4, 5, and 6) the left ventricle becomes more a cone than in diastole. In severe cases (Cases 7 and 8) the partition of the left ventricle into many compartments becomes apparent. Two patients (Cases IO and II) have a broken longitudinal axis; a third (Case I2) shows the same effect. In 2 patients (Cases 9 and I2) the development of multiple compartments is not striking. In systole I patient (Case 13) shows an exaggeration of the severe deformation of the left ventricular shape.

Electrocardiograms All patients were in sinus rhythm. The PR interval was prolonged $(>0.2 \mathrm{sec}$ ) in 4 cases and shorter than $0.13 \mathrm{sec}$ in $I$ case. The PR interval in the other 20 patients was within the normal range. In 4 patients the QRS time exceeded O. Io sec. One of these had a tracing similar to a left bundle-branch block. In 9 patients the electrocardiograms fulfilled the criteria for left ventricular hypertrophy $\left(S V_{I}+R V_{5}=3.5 \mathrm{mV}\right)$. Signs of right ventricular hypertrophy $\left(R V_{I}+R_{5}=I \cdot 05\right.$ $\mathrm{mV}$ ) were found in 12 cases. In 5 cases there were signs of biventricular hypertrophy (Table ). Pathological $\mathbf{Q}$ waves in chest leads $\mathrm{V}_{3}-\mathrm{V}_{7}$ were found in ro cases. The $\mathrm{T}$ waves in these leads were upright in all cases. Delta waves were found in chest leads within $\mathrm{V}_{4}-$ $\mathrm{V}_{7}$ in 6 patients; 5 more patients had delta waves in other leads. The $T$ waves of QRS complexes beginning with a delta wave were inverted in all cases. $Q$ waves and delta waves corresponded in the frontal plane in 2 cases (Fig. 2 and Table, Case I; Table, Case 3), and the horizontal plane in I case (Fig. 3 and Table, Case 9). The initial vector in an oesophagus lead was found to correspond with the initial vector of the opposite chest lead; this means a pathological $Q$ wave in a chest lead corresponded to a delta wave in an oesophagus lead of respective height and vice versa (see electrocardiogram, Fig. 2, delta wave E 30-Q V5/V6, Case I; Fig. 3, Q E 35 - delta wave V5/V6, Case 9; Fig. 4, Q E 40E 30 - delta wave V5/V6, Case 8).

Though the initial deflection of the ventricular complex occurs normally coincidentally in the various chest leads, this was not found in some patients with irregular hypertrophic cardiomyopathy. In I patient (Case I) (Fig. 2) the $\mathrm{Q}$ wave in $\mathrm{E}_{55}, \mathrm{~V}_{4}, \mathrm{~V}_{5}, \mathrm{~V} 6$, preceded the $R$ wave in $V_{1}$ and $V_{3}$ by 0.01 sec. 


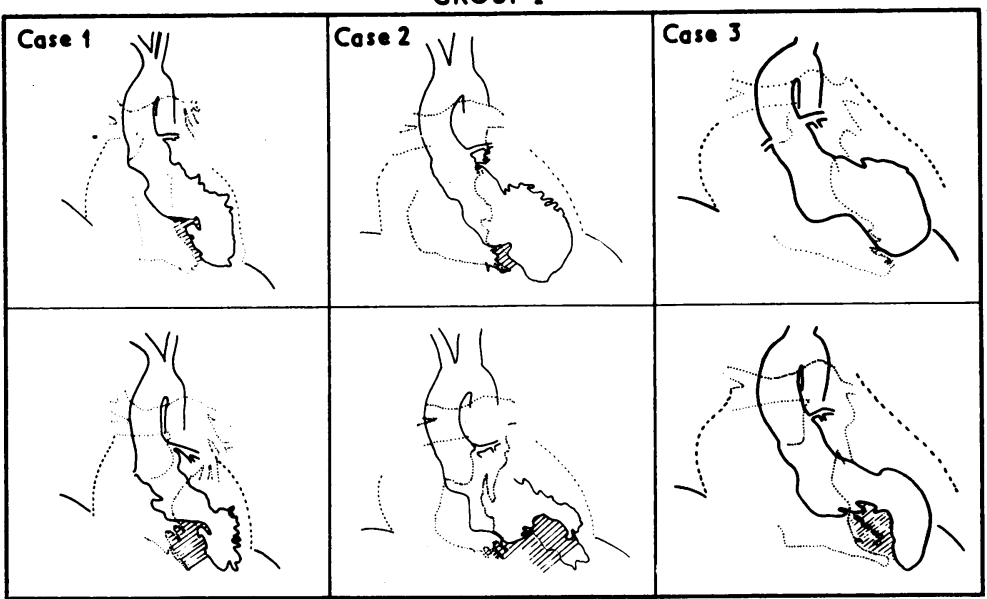

GROUP II a

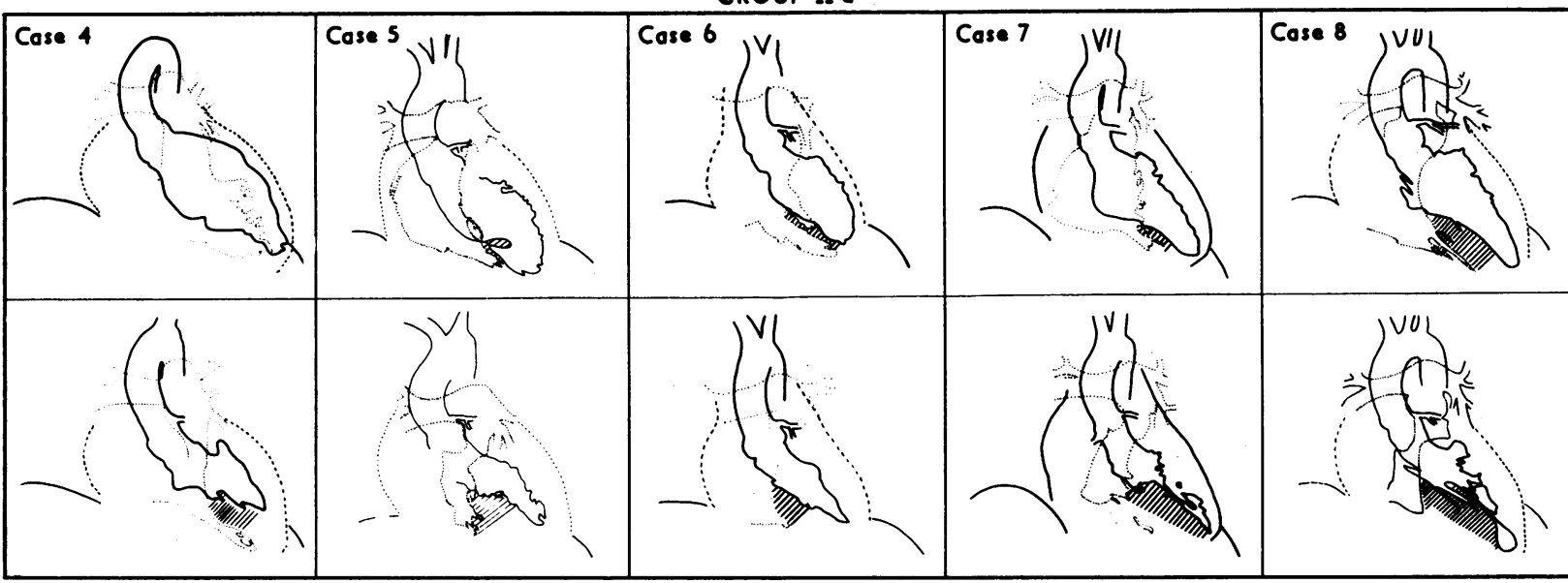

GROUP II b

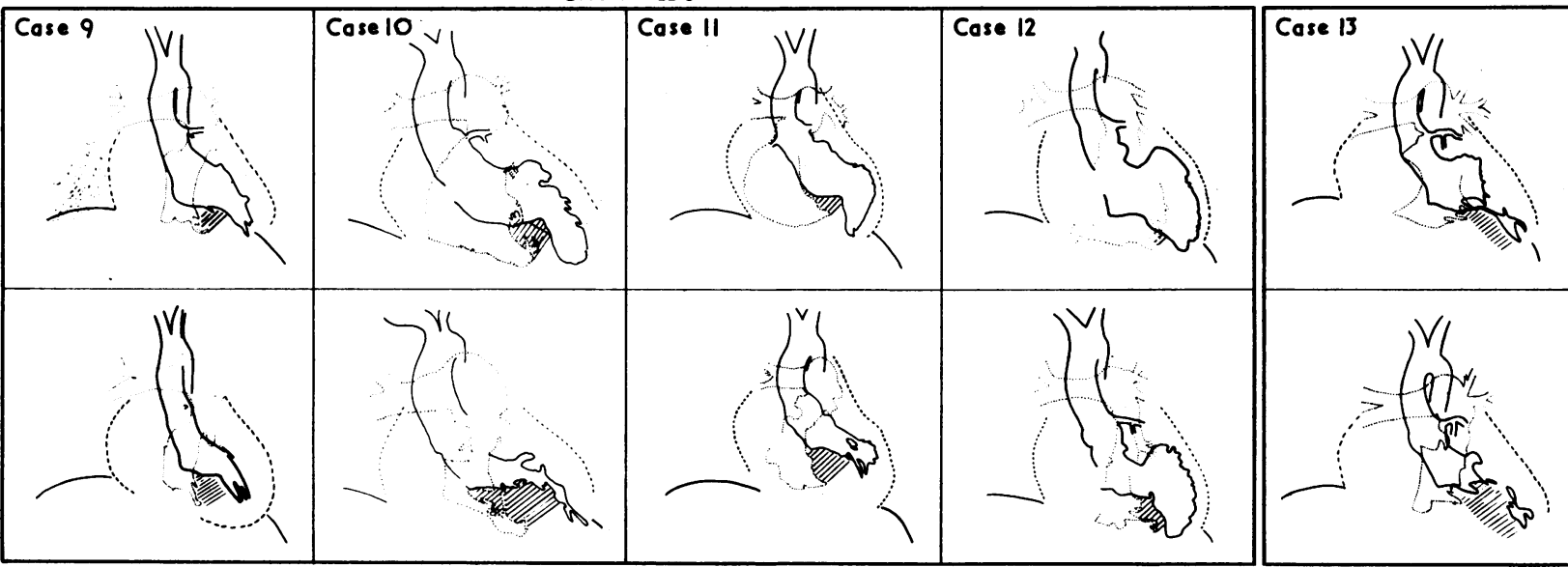

FIG. I Superimposed outlines of the right and left ventricular angiocardiograms of 13 patients. Upper Fig. in diastole, lower Fig. in systole. Hatched lines = the intraventricular septum. Roman numerals give the angiocardiographic group (see text). 

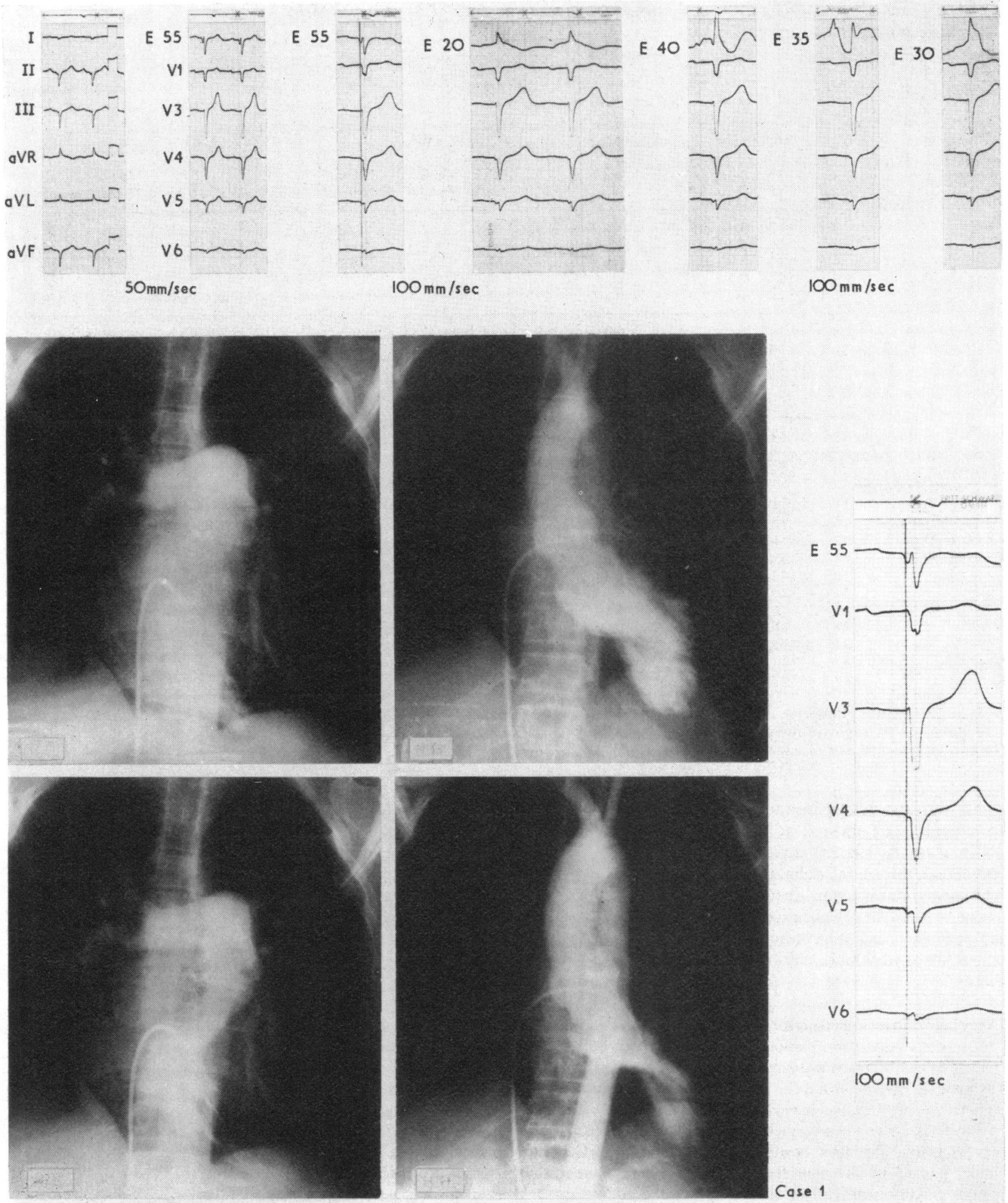

FIG. 2 Electrocardiogram of Case I with oesophageal leads. Pre-excitation is apparent in V4-V6 in the magnified Figure. In the angiocardiogram this patient showed circumscribed hypertrophy (Group I); upper left: right ventricle in diastole; upper right: left ventricle in diastole; lower left: right ventricle in systole; lower right: left ventricle in systole. For superimposed outlines see Fig. I, Case I. For oesophageal electrocardiogram, see text. 
TABLE The electrocardiographic and mechanocardiographic data of 25 patients with irregular hypertrophic cardiomyopathy

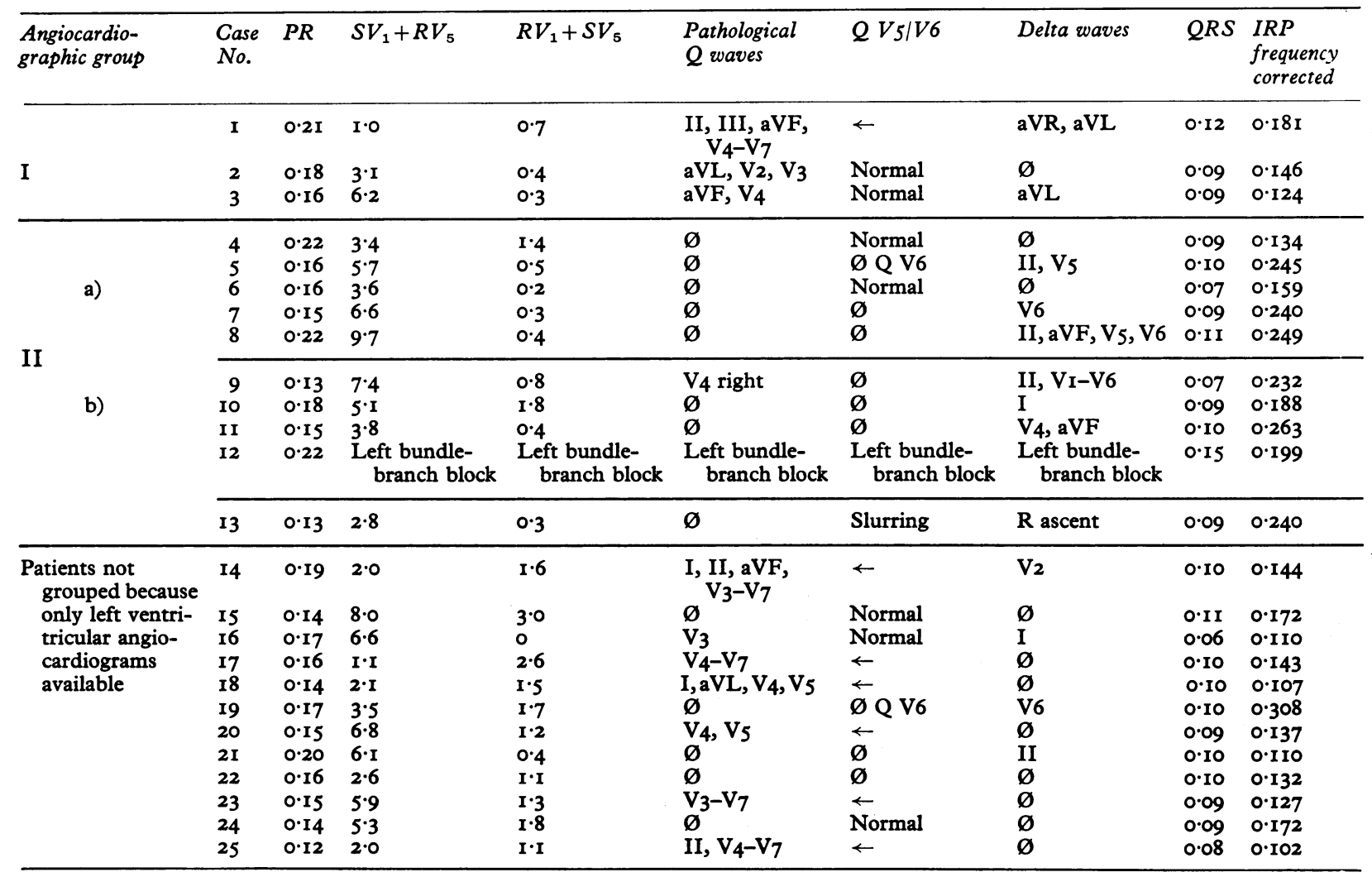

Angiocardiographic Group I = circumscribed hypertrophy of the ventricular septum. Angiocardiographic Group II = diffuse hypertrophy of the ventricular septum; IIa with straight longitudinal axis of left ventricle; IIb with broken longitudinal axis of left ventricle.

This was also found in another patient (Case 2) where $\mathrm{QV}_{3}$ and $\mathrm{r} \mathrm{E} 30$ preceded RVI.

In Case 8 (Fig. 4) delta waves in $\mathrm{V}_{3}-\mathrm{V}_{4}$ preceded the initial deflection of $\mathrm{VI}, \mathrm{V} 6$, and the oesophageal leads. In Case 9 delta waves were present in all chest leads (Fig. 3). Case I3 exhibits a peculiar slurring $R$ ascent up to the peak of $R$, which may be a variant of delta wave.

Mechanocardiographic results The isovolumetric relaxation period (IRP) taken from the apex cardiogram was prolonged in $2 \mathrm{I}$ out of 25 cases (Fig. 5, Table). The normal range in our laboratory for isovolumetric relaxation period is $0.105 \pm 0.014 \mathrm{sec}$ based on 60 cycles/min. The first component of the first heart sound in the low frequency band was phonocardiographically apparent and of considerable amplitude in patients with delta waves in leads V5 and V6, whereas this component was not visible or was faint in patients with pathological $Q$ waves in the chest leads.
Correlations At the time of ventricular depolarization the actual state of ventricular contraction is that of end diastole. Hence, the form of the ventricular septum in diastole was evaluated with the first vector of QRS. It was of further interest how circumscribed or diffuse hypertrophy influences the ventricular compliance as measured by the ventricular relaxation period.

Patients with delta waves in chest leads $\mathrm{V}_{4}-\mathrm{V}_{7}$ (Cases 5, 7, 8, 9, II, and I3) have a conspicuous prolongation of the isovolumetric relaxation period (Fig. 6). The form of the intraventricular septum is determined by diffuse myocardial hypertrophy (Group II). One patient (Case ro) neither has normal septal $Q$ waves in $V_{5} / \mathrm{V} 6$ nor delta waves in these leads. The isovolumetric relaxation period is shorter than in the cases with delta waves. The shortest values for isovolumetric relaxation period in group II are linked to two patients (Cases 4 and 6) whose electrocardiograms exhibit normal septal $Q$ waves and no delta 

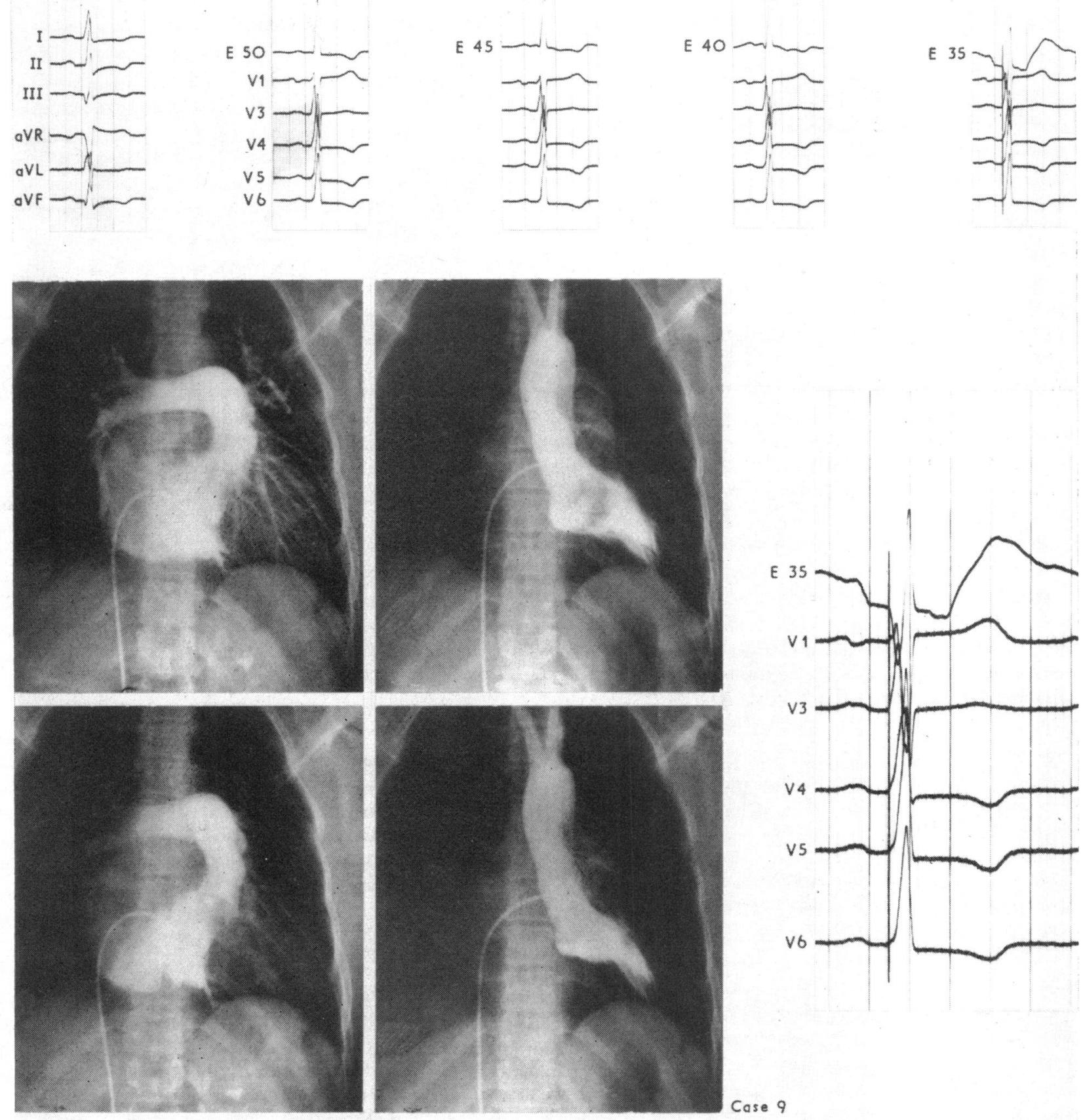

FIG. 3 Electrocardiogram of Case 9 (paper speed $100 \mathrm{~mm} / \mathrm{sec}$ ). Delta waves in VI, V4-V6 suggest diffuse pre-excitation (magnified Fig.). For oesophageal leads, see text. In the angiocardiogram the form of the ventricles is influenced by diffuse hypertrophy of the ventricular septum. The longitudinal axis of the left ventricle is broken (Group IIb). For superimposed outlines see Fig. I, Case 9. 


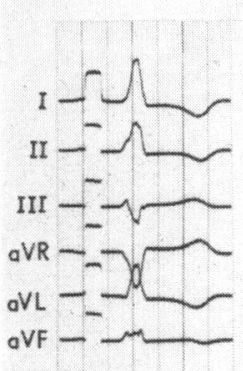

$100 \mathrm{~mm} / \mathrm{sec}$

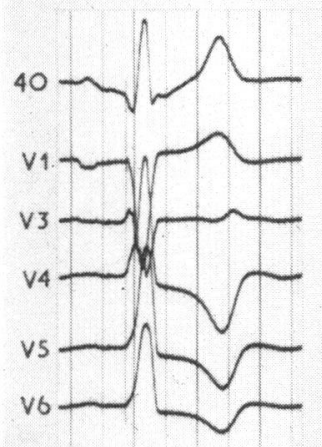

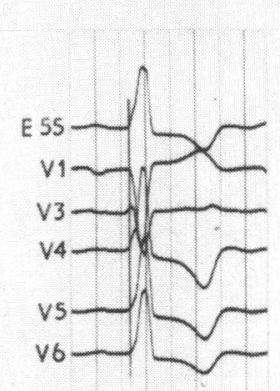
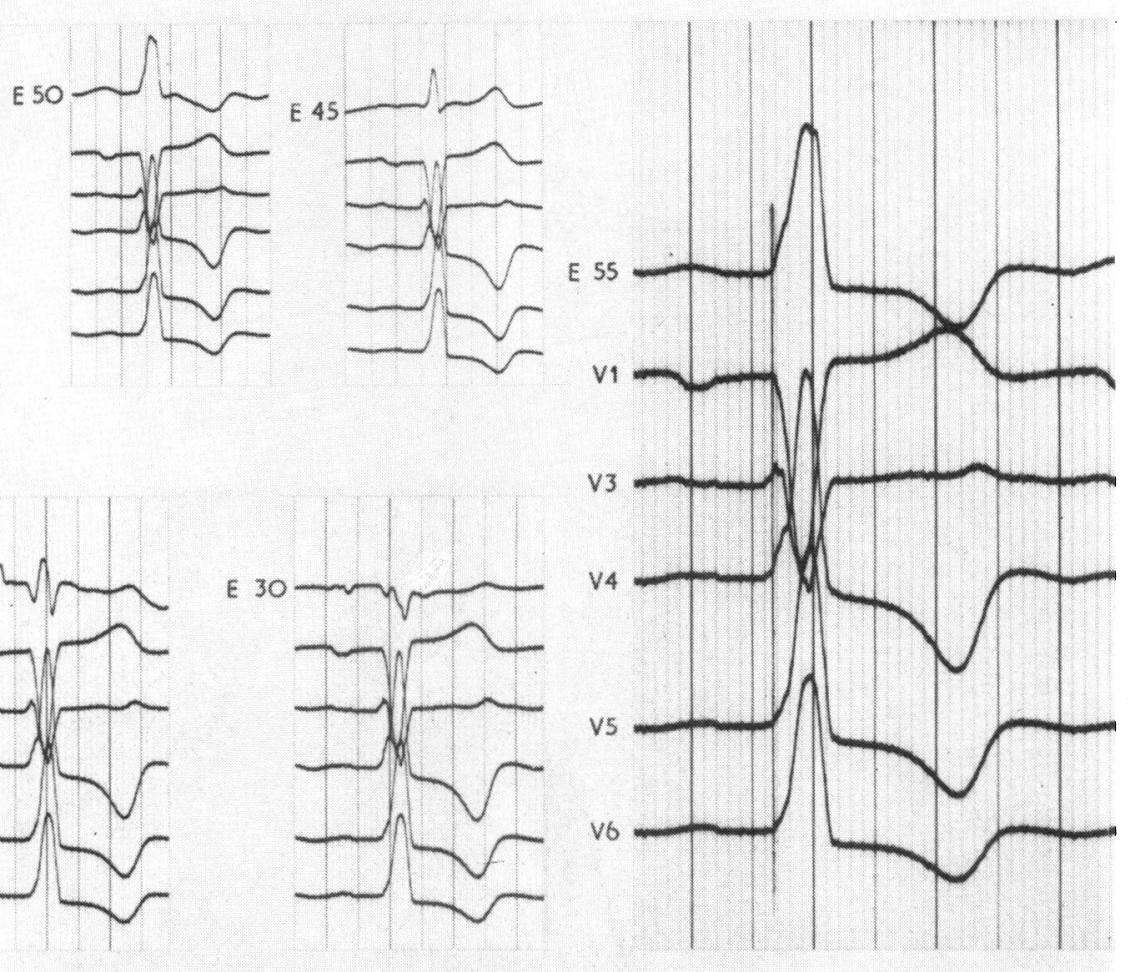

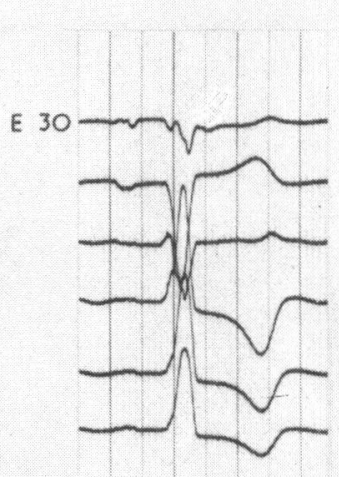

FIG. 4 Electrocardiogram of Case 8. The angiocardiogram shows diffuse septal hypertrophy and numerous indentations in the free left ventricular wall. Delta waves are apparent in V3-V6. Localized pre-excitation is seen in $V_{3}$ and $V_{4}$.

waves. Their angiocardiograms show moderate deformation of the ventricular chambers only.

In contrast to patients with delta waves,

FI G. 5 Apex cardiogram and phonocardiogram of a patient with irregular hypertrophic cardiomyopathy. The II-O time represents the isovolumetric relaxation time

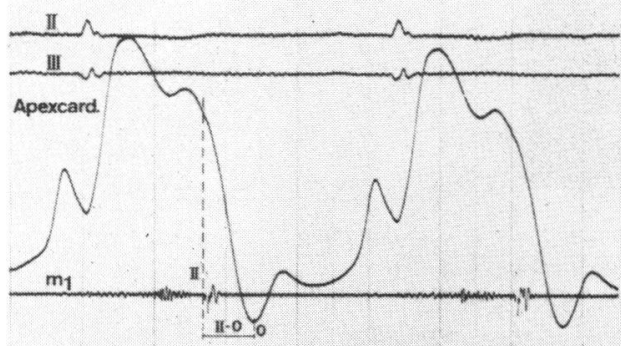

$m_{2}$

h those who exhibit pathological $Q$ waves in chest leads VI-V6 have only a slight prolongation of the isovolumetric relaxation period. The angiocardiogram reveals the ventricular septum to be locally hypertrophied (Group I). A slight prolongation of the isovolumetric relaxation period combined with pathological $Q$ waves also occurred in other cases (Fig. 6) not grouped because a left ventricular angiocardiogram only was available.

\section{Discussion}

Various authors have tried to correlate different parameters of this cardiomyopathy (Braunwald et al., 1964; Klein et al., 1968; Estes et al., 1963; Coyne, 1968). The pressure gradients were of special interest. However, no conclusive correlation resulted. The reason for this might be the beat-to-beat variability (Braunwald et al., 1964; Menges et al., 196I; Gorlin et al., 1964) of the pressure gradients. Our own experiences with this parameter confirm this.

Our assumption was that hypertrophy of 


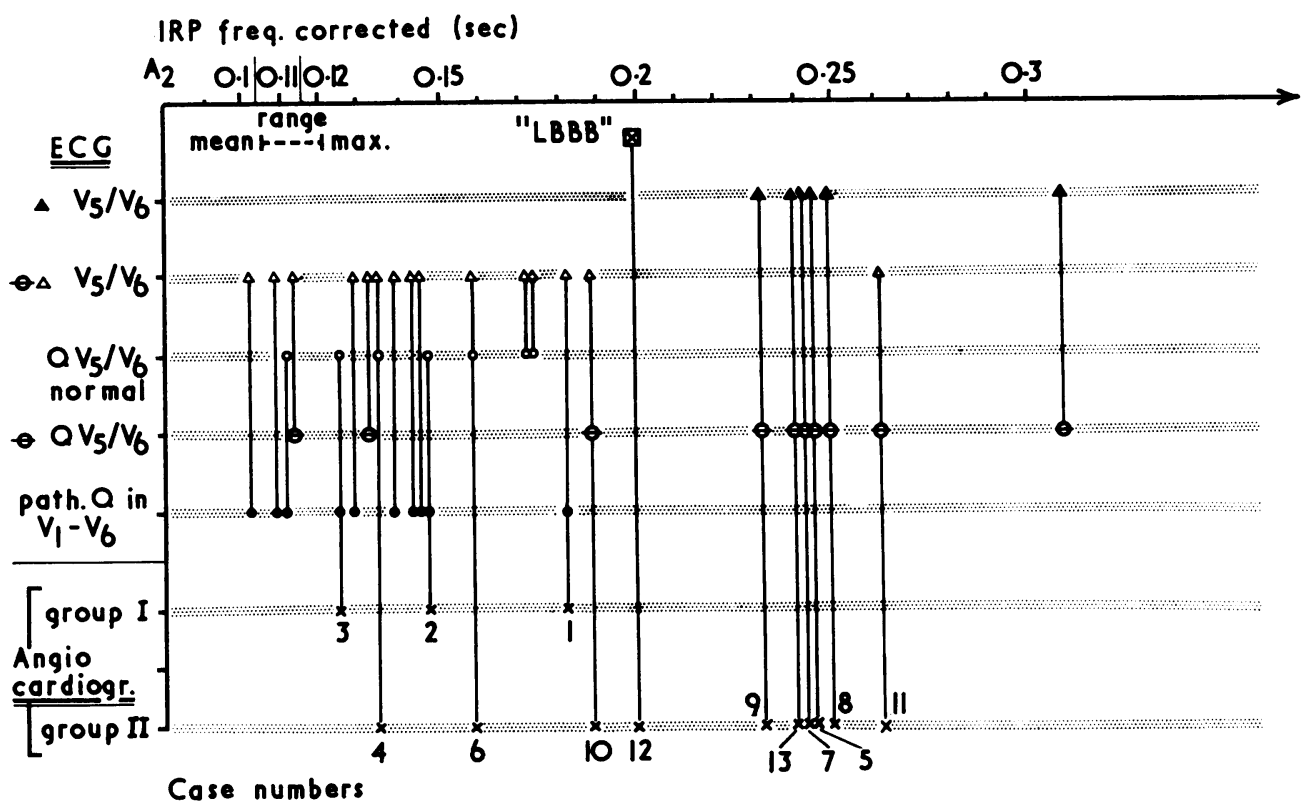

FIG. 6 Correlation of three parameters: the isovolumetric relaxation period (IRP), the first vector in the chest leads, and the form of the ventricular septum in diastole.

Electrocardiogram: $I R P=i$ sovolumetric relaxation period taken from the apex cardiogram; $\Delta V_{5} / V 6=$ delta waves in $V_{5} / V 6$ present; $\theta \triangle V_{5} / V 6=$ no delta waves in $V_{5} / V 6$ present; $Q V 5 / V 6$ norm $=Q$ wave in V5/V6 normal; $\theta Q V 5 / V 6=$ absence of $Q$ wave in $V 5 / V 6$; path. $Q$ in $V_{I}-V 6=$ pathological $Q$ wave in any lead $V_{I}-V 6$ present. Angiocardiogram: group I=predominant circumscribed hypertrophy of the ventricular septum; group $I I=$ predominant diffuse hypertrophy of the ventricular septum.The IRP is prolonged in all cases. In cases of Group I and those with moderate deformation of the ventricles of Group II (Cases 4 and 6) the IRP is less prolonged than in the cases of Group II with severe deformation. A pathological $Q$ wave is found only in cases with predominantly circumscribed septal hypertrophy (Group I). In Group II normal septal $Q$ waves disappear and become replaced by delta waves as the IRP increases.

the ventricular septum and adjacent myocardium causes a loss in compliance. This assumption seems to be proved, because the cases with diffuse septal hypertrophy have a significant longer isovolumetric relaxation period than those with circumscribed septal hypertrophy. The electrocardiogram and the apex cardiogram of 3 patients reported by Lucardie (1964) show the same but not described effect. Previously we found diffuse hypertrophy connected with delta waves. Pathological $Q$ waves were apparent in cases with circumscribed hypertrophy (Harmjanz et al., 1967a).

Beside the high intramural pressure in systole the loss in compliance may be of equal importance for the function of this disabled myocardium.

After finding that circumscribed hypertrophy was connected with pathological $\mathrm{Q}$ waves we tried to give graphically an explanation for the unique pattern (Fig. 7). We subtracted a
FIG. 7 Graphical subtraction of a normal $Q R S V_{3}$ pattern from an original $V_{3}$ pattern found in a patient with circumscribed hypertrophy of the septum. The resultant potential represents the excitation of the additional muscle mass remote from the electrode.

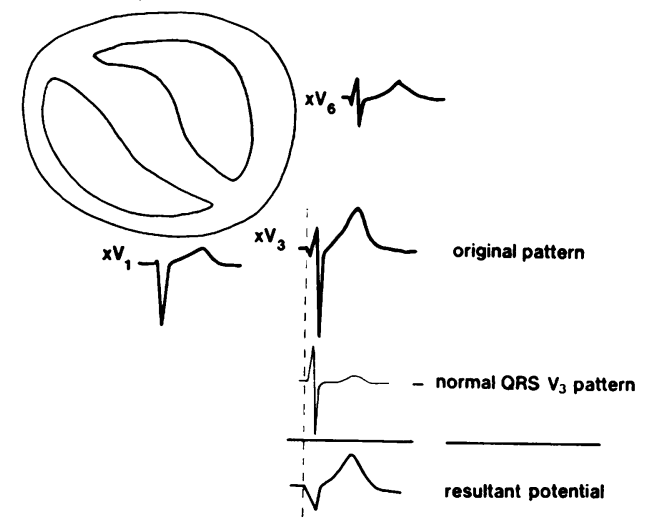


'normal' $V_{3}$ QRS pattern from a $V_{3}$ pattern found in a patient. The resultant pattern indicates that the initial vector moves in the direction of the circumscribed hypertrophied muscle mass. The $T$ wave is discordant, indicating disturbed repolarization.

The graphical derivation was performed with a V6 pattern of a patient with a delta wave in this lead (Fig. 8). Delta waves indicate the same mechanism as $Q$ waves, but are inverse. The vector of the additional early potential (delta waves) moves in the direction to the electrode. The result is a higher positive voltage. The repolarization phase generally shows ST depression. The accentuation of the first component of the first heart sound in patients with delta waves may reveal the widely spread hypertrophied myocardium as this component represents the acceleration of the blood before the atrioventricular valves are closed.

Snellen (1964) found an early excitation of the anterior paraseptal basal region (35-50 $\mathrm{msec}$ ) between operations (normal 45-50 msec). This part slightly preceded the activation of the apical region instead of following it. Snellen reported that the conduction time was increased to about $50 \mathrm{~cm} / \mathrm{sec}$ (normal 35 $\mathrm{cm} / \mathrm{sec}$ ). This result may throw light on our finding of early septal activation as suggested by the unique $Q$ waves and delta waves. In fact, these $Q$ waves as well as the delta waves precede the $R$ wave of other leads. Normally, the first deflection, positive or negative, occurs coincidently in all chest leads; in this light the finding of local pre-excitation deserves discussion.

Pre-excitation, usually accepted as being synonymous with WPW syndrome, covers a wider range than is generally accepted. All the classical features of WPW syndrome (PR shortening, slurring $R$ wave, prolonged $Q R S$ complex) were not present in our cases. However, one may consider the $Q$ waves and delta waves as representative of an incomplete WPW syndrome. The point against this assumption is that in the WPW syndrome the first deflection occurs synchronously in all chest leads whereas in irregular hypertrophic cardiomyopathy there is only a local preexcitation of the hypertrophied ventricular regions. This indicates a bypass below the AV node. In cases with widely diffuse hypertrophy of the ventricular myocardium, as in Case 9, the delta waves found in all chest leads may suggest general pre-excitation and thus these tracings become indistinguishable from incomplete WPW syndrome; the same effect may be responsible for a slurring $R$ ascent in Case I3.

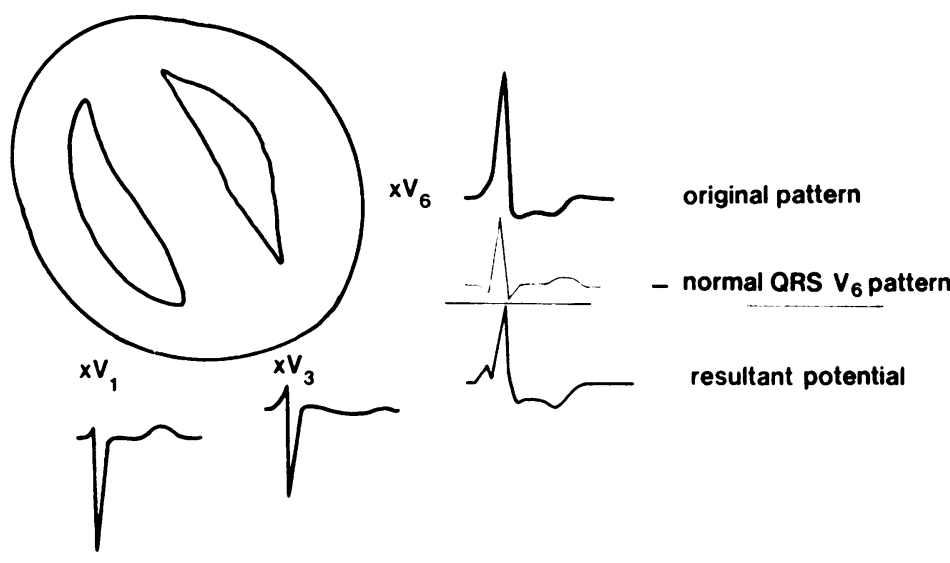

FIG. 8 Graphical subtraction of a normal $Q R S V 6$ pattern from an original pattern found in a patient with diffuse hypertrophy of the septum. The first vector runs in the direction of the electrode indicating an early excitation of the anterolateral portion of the left ventricular wall.

As we could show local pre-excitation, a focal block does not seem to be the cause of a delta wave in irregular hypertrophic cardiomyopathy (Coyne, 1968). Our patient (Case 12) with a left bundle-branch-block pattern may represent a diffuse pre-excitation.

Further studies with His bundle recordings should be done to show that the conduction in the AV node is unaltered in these patients.

Following the finding of Snellen our conclusion is that the hypertrophic myocardium in this special cardiomyopathy has a higher conductivity causing pre-excitation of the hypertrophic area. The representatives of that pre-excitation are the $Q$ waves and delta waves.

\section{References}

Braunwald, E., Lambrew, C. T., Rockoff, S. D., Ross, J., Jr., and Morrow, A. G. (I964). Idiopathic hypertrophic subaortic stenosis: I. A description of the disease based upon an analysis of 64 patients. Circulation, 30, Suppl. 4, p. 3.

Coyne, J. J. (1968). New concepts of intramural myocardial conduction in hypertrophic obstructive cardiomyopathy. British Heart fournal, 30, 546.

Estes, E. H., Jr., Whalen, R. E., Roberts, S. R., Jr., and McIntosh, H. D. (1963). The electrocardiographic and vectorcardiographic findings in idiopathic hypertrophic subaortic stenosis. American Heart fournal, 65, I55.

Gorlin, R., Cohen, L. S., Elliott, W. C., Klein, M. D., and Lane, F. J. (1964). Haemodynamics of muscular subaortic stenosis (obstructive cardiomyopathy). In Cardiomyopathies: Ciba Foundation Symposium, p. 76. Little, Brown, Boston. 
Harmjanz, D., Heimburg, P., Kochsiek, K., and Emmrich, J. (1967a). Vergleichende Untersuchungen zwischen Elektrokardiogramm und Angiokardiogramm bei irregulär hypertrophischer Kardiomyopathie. Zeitschrift für kreislaufforschung, 56, 580.

Harmjanz, D., Kochsiek, K., Heimburg, P., and Emmrich, J. (1967b). Auswirkungen der irregulär hypertrophischen Kardiomyopathie auf die Funktion und Form des rechten und linken Ventrikels. Zeitschrift für Kreislaufforschung, 56, 567.

Klein, M. D., Mathur, V., Levine, H. D., and Gorlin, R. (1968). Electromechanical correlations in hypertrophic subaortic stenosis. Circulation, 38, 635.

Lucardie, S. M. (1964). Phonocardiographical aspects of obstructive cardiomyopathy. Acta Cardiologica, I9, 478 .
Menges, H., Jr., Brandenburg, R. O., and Brown, A. L., Jr. (1961). The clinical, hemodynamic and pathologic diagnosis of muscular subvalvular aortic stenosis. Circulation, 24, I 126.

Ross, J., Jr., Braunwald, E., Gault, J. H., Mason, D. T., and Morrow, A. G. (1966). The mechanism of the intraventricular pressure gradient in idiopathic hypertrophic subaortic stenosis. Circulation, $34,558$.

Snellen, H. A. (1964). Diagnosis of various types of hypertrophic obstructive cardiomyopathy. In Cardiomyopathies: Ciba Foundation Symposium, p. 43. Little, Brown, Boston.

Requests for reprints to Dr. D. Harmjanz, 3 Hannover, Medizinische Hochschule, Oststadtkrankenhaus, West Germany. 